Draft VERSiON MARCH 6, 2018

Preprint typeset using $\mathrm{LATE}_{\mathrm{E}} \mathrm{X}$ style emulateapj v. 5/2/11

\title{
FROM BINARIES TO MULTIPLES II: HIERARCHICAL MULTIPLICITY OF F AND G DWARFS
}

\author{
ANDREI TOKOVININ \\ Cerro Tololo Inter-American Observatory, Casilla 603, La Serena, Chile \\ Draft version March 6, 2018
}

\begin{abstract}
Statistics of hierarchical multiplicity among solar-type dwarfs are studied using the distance-limited sample of 4847 targets presented in the accompanying Paper I. Known facts about binaries (multiplicity fraction 0.46 , log-normal period distribution with median period $100 \mathrm{yr}$ and logarithmic dispersion 2.4 , and nearly uniform mass-ratio distribution independent of the period) are confirmed with a high statistical significance. The fraction of hierarchies with three or more components is $0.13 \pm 0.01$, the fractions of targets with $n=1,2,3, \ldots$ components are 54:33:8:4:1. Sub-systems in the secondary components are almost as frequent as in the primary components, but in half of such cases both inner pairs are present. The high frequency of those $2+2$ hierarchies $(4 \%)$ suggests that both inner pairs were formed by a common process. The statistics of hierarchies can be reproduced by simulations, assuming that the field is a mixture coming from binary-rich and binary-poor environments. Periods of the outer and inner binaries are selected recursively from the same log-normal distribution, subject to the stability constraint and accounting for the correlation between inner sub-systems. The simulator can be used to evaluate the frequency of multiple systems with specified parameters. However, it does not reproduce the observed excess of inner periods shorter than $10 \mathrm{~d}$, caused by tidal evolution.

Subject headings: stars: binaries; stars: solar-type; stars: statistics
\end{abstract}

\section{INTRODUCTION}

Stars tend to be born in pairs and higher-order hierarchies. It has long been recognized that understanding stellar multiplicity is important in many areas of astronomy, from star formation to high-energy astrophysics. Here we address the statistics of hierarchical multiples (triple and quadruple systems) among solar-type dwarfs, using the data of Paper I (Tokovinin 2014).

Hierarchical stellar systems have been known since a long time. Batten (1973) highlighted their significance as a potential key to solving the mystery of binarystar formation (see recent discussion in Reipurth et al. 2014). Fekel (1981) published the first list of hierarchical triples. Meanwhile, other authors who studied binary statistics mentioned multiples only in passing. Typically small samples of $\sim 200$ stars in these early works contained only a few hierarchies.

The paper by Abt \& Levy (1976, AL76) demonstrated the importance of binary statistics and influenced the field for several decades. By a bold extrapolation it predicted that almost every solar-type star has a low-mass (planetary) companion. This prediction, shown later to be the result of data over-interpretation, stimulated nevertheless the search for very low-mass (VLM) companions and exo-planets.

The work by Duquennoy \& Mayor (1991, hereafter DM91) remains even today one of the most cited papers on binary statistics. They corrected the overly-optimistic prognosis of the frequency of VLM companions, while still focusing on their importance (few years later this team announced the first exo-planet around $51 \mathrm{Peg}$ ). A similar study of $\mathrm{K}$ and $\mathrm{M}$ dwarfs (Tokovinin 1992) reached the opposite conclusion that the VLM companions are rare; this is now confirmed and known as browndwarf desert. The log-normal distribution of periods pro-

atokovinin@ctio.noao.edu posed in DM91 was confirmed by further studies, including this one.

Two decades later, Raghavan et al. (2010, hereafter R10) revised the results of DM91 using better observing methods and a larger sample. They show that the mass ratio is distributed almost uniformly at all periods, overturning the claims to the contrary made in AL76 and DM91. The frequency of hierarchical systems was estimated at $f_{H}=12 \%$, doubled in comparison to DM91. We show below that it is even a little higher.

Most known multiple stars result from random discoveries. Information on triples and higher-order systems collected in the Multiple Star Catalog, MSC (Tokovinin 1997) is heavily distorted by observational selection and not suitable for unbiased statistical study. Tokovinin (2008) attempted comparative analysis of triples and quadruples based on the MSC. A similar effort was undertaken by Eggleton (2009), using bright stars and an ad hoc model of observational selection.

This work is based on the volume-limited sample of 4847 unevolved (or moderately evolved) stars within $67 \mathrm{pc}$ of the Sun with masses from 0.9 to $1.5 M_{\odot}$, presented in Paper I and called FG-67 sample hereafter. Its completeness is no less than $90 \%$ As hierarchical systems are less frequent than binaries, the large sample size and a good companion census are essential here. Equally important is the correction for the incomplete companion detection. Paper I outlines the model of companion's detection and provides estimates of detection probability (completeness) for each individual target, as a function of period and mass ratio. On average, $80 \%$ of companions to the main targets are detected. However, the census of sub-systems in the fainter, secondary components is

\footnotetext{
1 The range of primary masses in the FG-67 sample is less than in the 25-pc sample, so the number of targets in those samples does not scale as cube of their distance limits.
} 


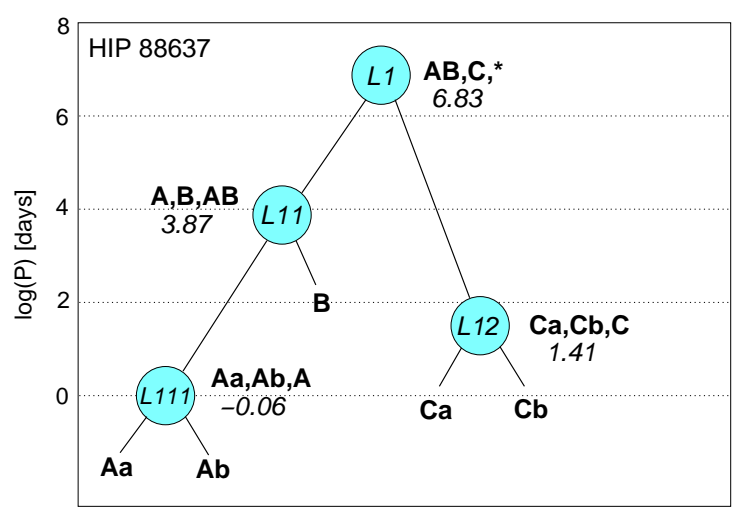

Fig. 1.- Hierarchical levels and companion designations for the quintuple system HIP 88637 are represented by a mobile diagram where vertical position of each sub-system reflects its period $x=$ $\log _{10} P / 1 \mathrm{~d}$. The hierarchy is coded by designating each system as (primary, secondary, parent), asterisk meaning the root. Levels are computed automatically from such designations. The outermost pair $\mathrm{AB}, \mathrm{C}, *(x=6.83)$ is at the root of the hierarchy, at L1. The sub-system in its primary component $\mathrm{A}, \mathrm{B}, \mathrm{AB}(x=3.87)$ is at $\mathrm{L} 11$, the secondary sub-system $\mathrm{Ca}, \mathrm{Cb}, \mathrm{C}$ is at $\mathrm{L} 12$, and the innermost binary $\mathrm{Aa}, \mathrm{Ab}, \mathrm{A}$ is at L111.

worse, about $30 \%$.

We begin with the definition of hierarchical levels and mass ratio in $\S 2$. Then in $\S 3$ the joint distribution of period and mass ratio is derived. In $\S$ the statistical model of hierarchies is developed. It is based on the simple idea that all sub-systems are randomly drawn from the same generating distribution of periods, subject only to the dynamical stability constraint. This recipe requires, however, some adjustments to match the data. The summary and discussion in $\S 5$ and $\S 6$, respectively, close the paper.

\section{DESCRIPTION OF HIERARCHICAL SYSTEMS}

\subsection{Definition of a hierarchical system}

A system of three or more bodies with comparable separations is dynamically unstable (Harrington 1972). In contrast, hierarchical multiples survive for a long time because the motion in the close (inner) pair is not strongly perturbed by the outer companion(s). Stellar motions in stable hierarchies are represented approximately by Keplerian orbits. This is the definition of a hierarchical multiple system. This definition is not very sharp because some systems approach the stability boundary. Moreover, the orbital motion of very wide binaries is not observable owing to their long periods, so the borderline between the truly bound multiple systems and other stellar groups like disintegrating clusters is fuzzy.

\subsection{Hierarchical levels and multiplicity fractions}

Hierarchies can be described by binary graphs or trees, also called mobile diagrams (one example is shown in Figure 1). The position of each pair in the tree is called level and is denoted here as L1, L11, etc. The outermost (widest) pair is at the root of the tree, L1 (the root is denoted by asterisk in the parent designation). Inner pairs associated with the primary and secondary components are at levels L11 and L12, respectively, and this notation continues to deeper levels. Triple systems can have inner pair either at L11 or at L12. When both primary and secondary sub-systems are present, we get the so-called 2+2 system 2 Alternatively, a $3+1$ quadruple system consists of levels L1, L11, and L111; it has three companions associated with the same primary star, resembling in this respect a planetary system. Both types of hierarchy are found in HIP 88637. The assignment of "primary" and "secondary" is traditionally based on the apparent brightness of the components. When they are comparable in brightness or mass, the distinction between primary and secondary becomes irrelevant.

While we deal with binary and triple systems, their hierarchy is uniquely defined by the number of components, so levels are almost redundant. On the other hand, quadruple and higher-order systems can have different organizations, so their hierarchy requires additional descriptors such as levels. From the observational point of view, it is easier to explore lower hierarchical levels L1, L11, and L12 than to determine the number of multiple systems with exactly 3 , 4, etc. components.

The fraction of non-single stellar systems, the multiplicity fraction $f_{M}$, equals the fraction of L1 systems in a given sample. The companion fraction $f_{C}$ is the number of systems at all levels divided by the sample size, so $f_{C}>f_{M}$. It is easier to measure $f_{M}$, as it does not depend on the discovery of all sub-systems. Multiplicity is also characterized by the fractions of systems with exactly $n$ components, $f_{n}$. Obviously, the fraction of single stars $f_{1}=1-f_{M}$, while $f_{C}=f_{2}+2 f_{3}+3 f_{4}+\ldots$ The fraction of hierarchies in a sample is $f_{H}=\sum_{n=3}^{\infty} f_{n}=$ $1-f_{1}-f_{2}$. The multiplicity statistics are defined by $f_{n}$, by the distribution of levels, and by the joint distributions of periods and mass ratios at different levels.

The system depicted in Figure 1 contains hierarchical levels most frequently found in the FG-67 sample: L11 $(N=296), \mathrm{L} 12(N=95)$, and L111 $(N=17)$. Here the statistical analysis of those three levels is made, because higher levels are rare, and a much larger sample would be needed for their study.

\subsection{Mass ratio definition}

In a binary system with the primary component $\mathrm{A}$ and the secondary component $\mathrm{B}$, the mass ratio is defined as $q=M_{B} / M_{A}<1$. In a triple system where $\mathrm{B}$ is a close pair $\mathrm{B}, \mathrm{C}$, the mass ratio can be defined in different ways (e.g. Tokovinin 2008). The classical approach is to use the mass sum of sub-systems in the system mass ratio $q_{\mathrm{sys}}=\left(M_{B}+M_{C}\right) / M_{A}$. However, this definition may result in $q_{\mathrm{sys}}>1$ even when the component $\mathrm{A}$ is the most massive and brightest star in the system. Alternatively, the component mass ratio $q=M_{B} / M_{A}$ takes into account only the masses of individual stars (primary components of respective sub-systems). For example, in a triple star consisting of the close binary A,B with a distant tertiary component $\mathrm{C}, q=M_{C} / M_{A}$, while $q_{\text {sys }}=M_{C} /\left(M_{A}+M_{B}\right)$. The detection limits of imaging techniques are based on the magnitude difference which is more tightly related to the component mass ratio $q$ than to $q_{\text {sys }}$. The use of $q$ avoids re-definition of "primary" and "secondary" that would be needed in many instances if we used the mass sum instead. In the following, we use consistently the component mass ratio $q$ for

2 A $2+2$ system can contain higher hierarchical levels, hence more than 4 stars, see Figure 1 In contrast, a $2+2$ quadruple contains exactly 4 stars. 

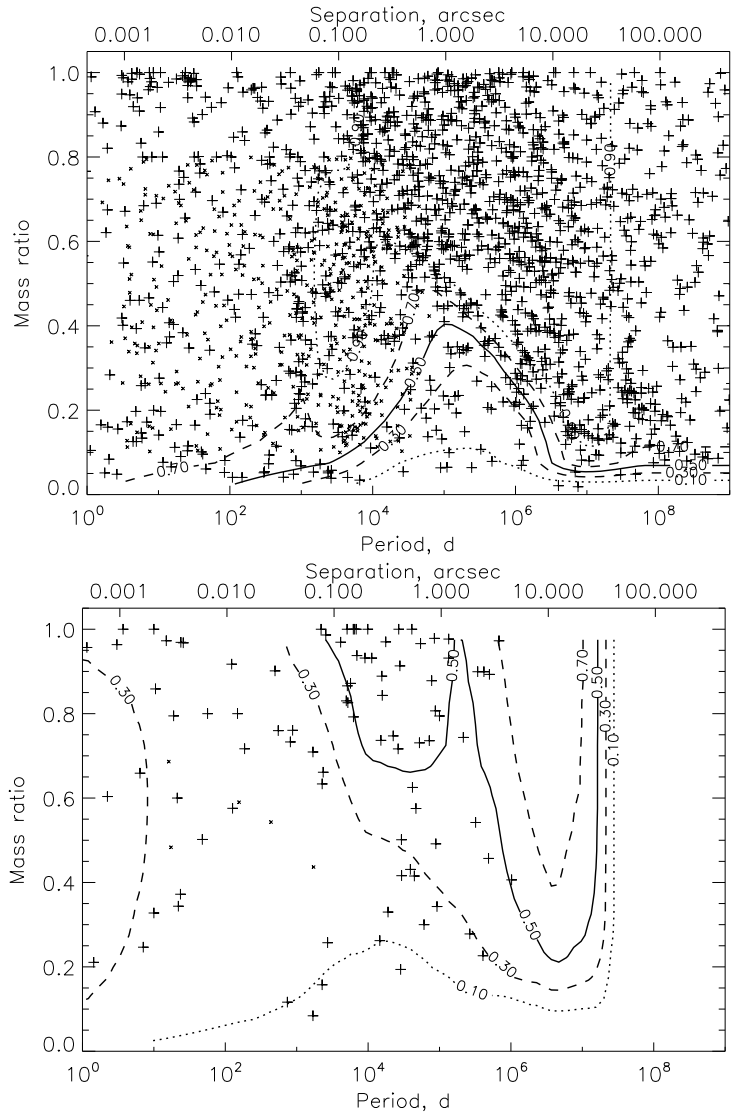

FIG. 2.- Distribution of systems around primary targets (top panel) and sub-systems in the secondary components (bottom panel $)$ in the $(P, q)$ plane. Plus signs - known $P, q$, small crosses - fictitious values. The contours indicate completeness of $0.1,0.3$, 0.5 (full line), 0.7 , and 0.9 . The upper axis corresponds to the angular separation at a distance of $50 \mathrm{pc}$.

multiple systems.

\subsection{Statistical data on the FG-67 sample}

The data on all systems and sub-systems in the FG-67 sample are assembled in the structure (table) which contains information on the hierarchical level (with links to parents and siblings), period, and component's masses. The same structure is produced for the simulated samples. As binary periods $P$ span a huge range, we use instead the logarithm $x=\log _{10}(P / 1 d)$ throughout this paper.

The 22 targets containing white dwarfs (WDs) are removed, leaving 2162 systems and sub-systems to consider (the sample excludes evolved primary components by design, WDs are such former primaries). The fraction of targets with WD companions is from $2 \%$ to $4 \%$, according to the rather uncertain estimate of Paper I. As only $0.5 \%$ of known binaries are removed and most of the remaining WDs are undiscovered, the total sample size without WDs should be somewhere between 4650 and 4750. The sample size affects the estimates of $f_{M}, f_{C}$, etc. Here we set it conservatively to 4800 (i.e. remove $1 \%$ of targets with WDs). The multiplicity fraction derived here would be larger by $\sim 1 \%$ if more targets with WDs were removed. Interestingly, among the 22 binaries with WDs, 11 are at least triple, their FG-type primaries (former secondaries) contain sub-systems. This supports the large fraction of secondary sub-systems found here in
TABLE 1

Statistical Data (Fragment). ${ }^{\text {a }}$

\begin{tabular}{cllccccc}
\hline \hline HIP0 & L & Type $^{\mathrm{b}}$ & $\begin{array}{r}x \\
\mathrm{~d}\end{array}$ & $\begin{array}{c}M_{1} \\
M_{\odot}\end{array}$ & $\begin{array}{c}M_{2} \\
M_{\odot}\end{array}$ & $\begin{array}{c}M_{1, p} \\
M_{\odot}\end{array}$ & $\begin{array}{l}M_{2, p} \\
M_{\odot}\end{array}$ \\
\hline 223 & 1 & $\mathrm{v}$ & 5.16 & 1.17 & 0.84 & 1.17 & 0.84 \\
290 & 1 & $\mathrm{~s}, \mathrm{a}$ & -9.00 & 1.28 & 0.00 & 1.28 & 0.00 \\
359 & 1 & $\mathrm{a}$ & -9.00 & 0.96 & 0.00 & 0.96 & 0.00 \\
394 & 1 & $\mathrm{~S} 1$ & 2.84 & 1.43 & 0.00 & 1.43 & 0.00 \\
493 & 1 & Cpm & 8.83 & 1.09 & 1.67 & 1.09 & 0.88 \\
493 & 12 & Ch & 5.62 & 0.88 & 0.79 & 0.88 & 0.79 \\
518 & 1 & $\mathrm{~V}$ & 4.59 & 1.06 & 1.37 & 1.06 & 0.91 \\
518 & 12 & S1 & 1.68 & 0.91 & 0.46 & 0.91 & 0.46 \\
522 & 1 & Chp & 5.37 & 2.25 & 0.54 & 1.20 & 0.54 \\
522 & 11 & v & 3.87 & 1.20 & 1.05 & 1.20 & 1.05 \\
\hline
\end{tabular}

a Table 1 is published in its entirety in the electronic edition of AJ, a portion is shown here for guidance regarding its form and content.

b Type means discovery techniques, e.g. s,S1,S2 - spectroscopic binaries, a, A - astrometric binaries, v, V - visual binaries, $\mathrm{C}$ - wide common proper motion pairs; see $\S 3$ of Paper I

the main sample.

The statistical data are presented in Table 1. Its columns contain the HIP), the Hipparcos number of the primary component, hierarchical level L, type of the system (see Paper I), logarithm of the period $x$ (in days), system masses of the primary and secondary components $M_{1}$ and $M_{2}$ (in solar mass), and the component masses $M_{1, p}$ and $M_{2, p}$ (primaries in the inner sub-systems). The two definitions of mass ratio in $\S 2.3$ correspond to $q_{\mathrm{sys}}=M_{2} / M_{1}$ and $q=M_{2, p} / M_{1, p}$. Masses are taken from the SYS table of Paper I, they are estimated from absolute magnitudes using standard relation for mainsequence stars. Unknown masses have zero values, unknown periods are assigned $x=-9$. The links to children and parent (record numbers in the data structure, or -1 if absent) are generated automatically using component designations; they are not printed in Table 1

A significant number, 355 out of 2162 systems, have unknown periods and separations. These are spectroscopic binaries without known orbit and binaries discovered by Hipparcos accelerations (see Paper I). Masses of their secondary components are also unknown. The statistical analysis presented below deals with this missing information (32\% of all binaries with periods shorter than $100 \mathrm{yr}$ ). The detection probability of these binaries is properly modeled, so we cannot simply ignore them. The assumption is made that all unknown periods are shorter than $\sim 100 \mathrm{yr}$. Random fictitious periods are generated to replace missing data when plotting period distributions, but these fictitious data are not used in any other way. Considering the detection limits of spectroscopy and astrometry, fictitious periods of spectroscopic binaries are uniformly distributed between $0.5<x<3.2$, acceleration binaries with constant radial velocity have $3.2<x<4.5$, e.g, HIP 290 and HIP 359 in Table 1. Partially missing information is the weakness of the FG-67 sample that affects the statistics at periods shorter than $100 \mathrm{yr}$.

Figure 2 shows position of known companions to primary targets and to the secondaries in the $(P, q)$ plane. Binaries with unknown parameters are plotted by small symbols to indicate their existence and likely location in this diagram. The contours show average detection 


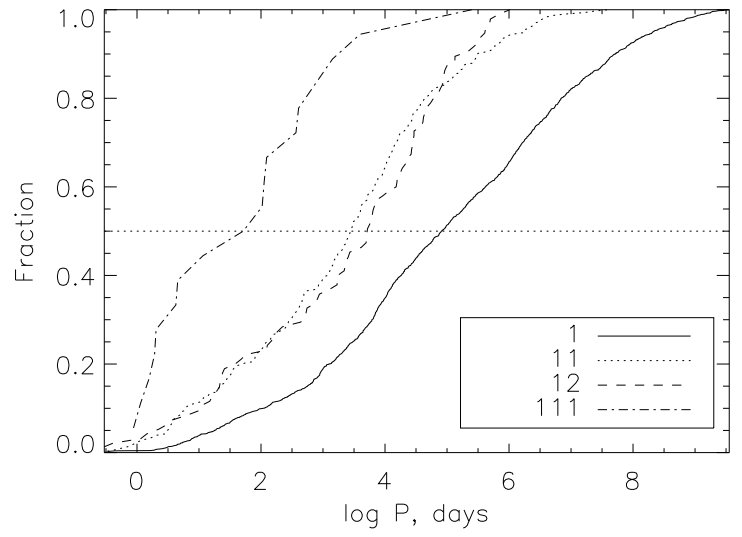

FIG. 3.- Cumulative histogram of periods at various hierarchical levels. Unknown periods are complemented by fictitious values.

probability. The census of low-mass companions with separations from $0^{\prime \prime} 3$ to $10^{\prime \prime}$ is still rather incomplete, other regions of the parameter space are covered better. The mass ratios are distributed almost uniformly at all periods. The period distribution has a broad maximum and declines on both sides of it. Note the cluster of twin binaries with $q \approx 1$ at $P<100 \mathrm{~d}$ (Tokovinin 2000; Lucy 2006). Figure 3 shows cumulative distributions of periods at three hierarchical levels (with fictitious periods included).

\section{DISTRIBUTION OF PERIOD AND MASS RATIO}

At any given period, the completeness of companion detection depends on the mass ratio. Therefore, the distributions of period and mass ratio must be determined jointly, taking into account the detection probability $d(x, q)$ for each target. Integrals of these distributions give the multiplicity fraction and $f_{n}$.

Our goal - the unbiased statistics of hierarchies - is approached by two complementary methods. First, we model the distribution of binaries in period and mass ratio $f(x, q)$ at various hierarchical levels by analytical functions and estimate the parameters of these models, accounting for the selection and the missing data (Tokovinin et al. 2006; Allen 2007). Second, a statistical model is developed by simulating the population of binary and multiple stars, simulating their incomplete detection, and comparing the result with the real sample. In this second approach, adopted e.g. by Eggleton (2009), the model has to be elaborated by trial and error. The simulated sample represents then the selectioncorrected statistics. The results of both methods are in mutual agreement.

\subsection{Analytical distribution of $P, q$}

Since Duquennoy \& Mayor (1991) fitted a log-normal function to the distribution of periods of 82 binaries, following the earlier work by Kuiper (1935), this analytical model has been universally adopted. The distribution of the mass ratio is often approximated by the power law $f(q) \propto q^{\beta}$ (Duchêne \& Kraus 2013). It is still being debated whether the exponent $\beta$ depends on period and mass or is universal (Reggiani \& Mever 2013). Following the tide, we represent the period distribution by the truncated log-normal law and the $q$ distribution by the power law with $\beta>-1$, truncated at $q<0.05$ (ignore

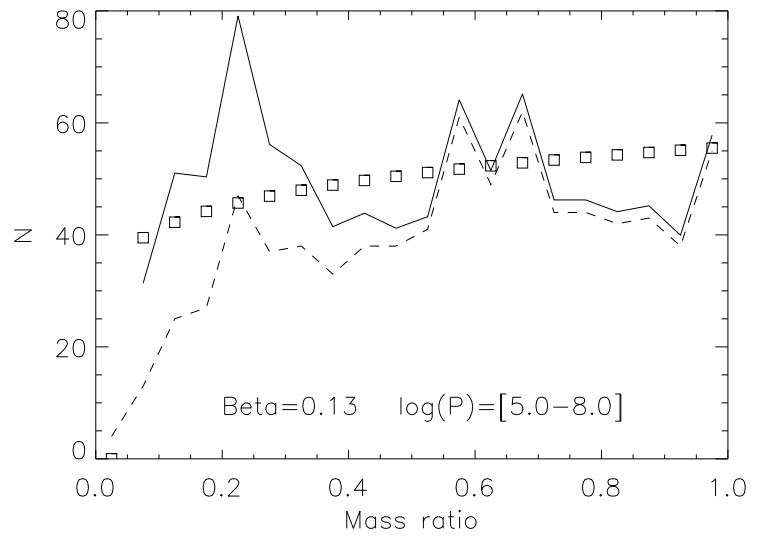

FIG. 4. - Histogram of mass ratio for 766 wide binaries with $5<x<8$. Dashed line: observed, full line: selection-corrected, squares: power law.

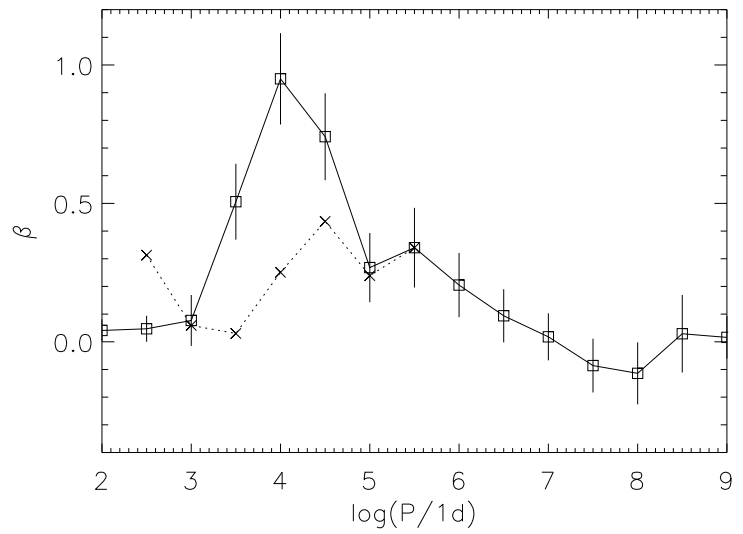

FIG. 5.- Dependence of the mass-ratio exponent $\beta$ on period. Each point corresponds to 1 dex interval of period. The crosses and dotted line show alternative calculation by complementing unknown periods and mass ratios with their plausible values.

brown-dwarf companions). The analytical model is

$$
f(x, q)=C \epsilon q^{\beta} \exp \left[-\left(x-x_{0}\right)^{2} /\left(2 \sigma^{2}\right)\right],
$$

where $x=\log _{10}(P / 1 d)$. The period distribution is truncated at $x<-0.3$ and $x>10$ and re-normalized accordingly by the constant $C$; without truncation, $C=$ $(1+\beta) /(\sigma \sqrt{2 \pi})$. The fitted parameters are multiplicity fraction $\epsilon$, median period $x_{0}$, period dispersion $\sigma$, and the mass-ratio exponent $\beta$. The assumption that $\beta$ is same at all periods is tested in the following sub-section. The correspondence between $\epsilon$ and $f_{M}$ or $f_{C}$ depends on the choice of binaries, see 33.3

The parameters of the model (1) were fitted to various sub-samples using maximum likelihood (ML) method. It takes into account the detection limits and deals with the missing data. The algorithm and its implementation are described in Appendix A.

\subsection{Dependence of mass ratio on period?}

Potential dependence of mass ratio on binary period can be studied here, taking advantage of the large sample size and known detection completeness. Binaries in certain period intervals were selected and fitted with just two parameters $(\epsilon, \beta)$ by the ML method (Appendix A). The detection probability $d(x, q)$ was averaged over these period intervals, leaving only its dependence on $q$. We 
TABLE 2

Fitted Distribution PARAMETERS

\begin{tabular}{lccccc}
\hline \hline Case & $N$ & $\epsilon$ & $x_{0}$ & $\sigma$ & $\beta$ \\
\hline All pairs & 2162 & 0.571 & 4.54 & 2.40 & 0.094 \\
& & \pm 0.012 & \pm 0.06 & \pm 0.06 & \pm 0.020 \\
L1 & \multirow{2}{*}{1747} & 0.464 & 4.93 & 2.34 & 0.051 \\
& & \pm 0.011 & \pm 0.06 & \pm 0.06 & \pm 0.011 \\
L11 & \multirow{2}{*}{296} & 0.214 & 3.25 & 1.80 & 0.121 \\
& & \pm 0.012 & \pm 0.12 & \pm 0.09 & \pm 0.026 \\
L12 & 95 & 0.157 & 2.67 & 1.68 & 1.32 \\
& & \pm 0.016 & \pm 0.17 & \pm 0.10 & \pm 0.28 \\
\hline
\end{tabular}

did not fit the histograms of $q$, avoiding any binning, but they match the derived $\beta$ reasonably well (Figure 4 ).

The results of such calculations in the overlapping period intervals of one decade are plotted in Figure 5. Each interval contains from 70 to 300 binaries. Only companions to the main targets are used here, regardless of their hierarchical level. The secondary sub-systems have different, smaller completeness, and are too few (95 total). At both short and long periods, the distribution of the mass ratio is uniform, with $\beta \approx 0$. This matches the results of other studies (Tokovinin 2011; Reggiani \& Mever 2013).

In the period interval $x=[3,5]$, the derived $\beta>0.5$ implies preference of large mass ratios. Indeed, most known binaries with such periods have $q>0.5$ because companions with $q<0.5$ have a low detection probability (see Figure 2). The detection probability is taken into account in the calculation of $\beta$; however, it ignores binary systems with unknown $P, q$, while including their discovery by acceleration and radial velocity in the evaluated completeness. To access the importance of this effect, we assigned fictitious periods and mass ratios to the binaries with missing data and repeated the calculation. The fictitious mass ratios are uniformly distributed in the interval [0.2-0.8] at periods shorter than $x=3.5$ and in the interval $[0.2,0.6]$ at periods $3.5<x<4.5$ (if such binaries had larger mass ratios, they would have been resolved). By complementing the missing data in this reasonable, but arbitrary way, we obtain smaller values of $\beta$ in the problematic period interval $3<x<5$ (dashed line and crosses in Figure 2). We conclude that the large values of $\beta$ likely result from the missing data and are not real.

There remains a hint on a mild dependence of $\beta$ on period even at $x>5$, where there are no missing data. However, the range of this variation, if any, is small. We fit the parameters of the model (1) by assuming a constant $\beta$. This helps to minimize the effect of missing information at intermediate periods. The global fitting in $\oint 3.3$ confirms the small values of $\beta$.

\subsection{Parameters of the distributions}

Table 2 lists the parameters of the distributions found by the ML method, for all pairs without distinction of their hierarchy and for the sub-samples. Its second column gives the number of binaries selected. The errors corresponding to $68 \%$ confidence intervals (" $1 \sigma$ ") are evaluated by the ML method. We checked that there is no strong correlation between parameters. However, one should keep in mind that these errors do not account for any problems in the input data or their modeling. The formal ML errors give a lower bound of the true errors.

When all pairs are selected, their fraction $\epsilon=0.57$ equals the companion fraction $f_{C}$. However, the code does not consider the reduced probability of detecting sub-systems in the secondary components, hence delivers the slightly under-estimated $f_{C} ; f_{C}=0.65$ follows from the simulations presented below in $\S 4.3$. When only the pairs at L1 (outer systems) are selected, we obtain the multiplicity fraction $\epsilon=f_{M}=0.46 \pm 0.01$. It agrees well with $f_{M}=0.46$ found by Raghavan et al. (2010) for solar-type stars.

For the sub-systems at levels L11 and L12, the presence of the outer pair at L1 is a necessary condition. Therefore, the resulting frequency $\epsilon$ refers to the sample of L1 systems, not to the whole sample. The ML results indicate that $0.464 \times 0.214=0.100$ fraction of all targets have at least two companions around their primary stars. Similarly, $0.464 \times 0.157=0.073$ fraction of targets have binary secondaries. This last estimate depends on the larger (and less certain) correction for the low probability of detecting secondary sub-systems.

The total fraction of hierarchical systems $f_{H}$ is less than the sum of the above numbers $(17 \%)$ because those two groups overlap (see below). About 0.6 fraction of L12 (secondary) sub-systems also have a L11 (primary) subsystem. The fraction of hierarchical systems is therefore estimated by ML as $f_{H}=0.100+0.4 \times 0.073=0.129$. A ballpark estimate of the uncertainty of this number is $\pm 1 \%$. Simulations presented in $\S 4.3$ confirm the derived fraction of hierarchies. Our initial analysis (before removing the WDs) gave $f_{H}=0.14$.

Figure 6] shows the period histograms of all pairs and of primary sub-systems at L11. Randomly assigned fictitious periods do not allow a rigorous comparison with the model in the $x<4.5$ zone. The incompleteness in each period bin is corrected by averaging $d(x, q)$ over $q$, which is valid for the uniform mass-ratio distribution only. Despite these shortcomings, the log-normal period distribution appears to be a good match to the data (the dotted curves were not fitted to the histograms!).

Previously, when the samples of binaries were small, the log-normal period distribution was an adequate model. However, its wings extend to unrealistically short and long periods; for this reason we use the truncated Gaussian here. A better analytic model of the period distribution could be a triangular function with a rounded top,

$$
f_{T}(x)=\frac{1}{C w}\left[1-\sqrt{a^{2}+\left(\left(x-x_{0}\right) / w\right)^{2}\left(1-a^{2}\right)}\right],
$$

where $\left|x-x_{0}\right|<w$. This distribution extends over finite range from $x_{0}-w$ to $x_{0}+w$, the parameter $w$ is close to the half-width. The parameter $a$ provides for the rounded top $(a=0$ means pure triangle $1-|x / w|)$, the normalization factor $C$ depends on $a$ : $C=1-a^{2}\left(1-a^{2}\right)^{-1 / 2} \ln \left[\left(1+\left(1-a^{2}\right)^{1 / 2}\right) / a\right]$. The need for three parameters $x_{0}, w, a$ instead of just two $x_{0}, \sigma$ may be a disadvantage of this model, but on the other hand the triangular distribution does not require truncation.

The ML formalism was adapted to use the triangular function instead of a Gaussian. The results obtained with this alternative model are quite similar, they are not given here for brevity. The parameter $a \approx 0.25$ was found, meaning that the top of the triangle is rounded only mildly. The "shoulders" of the period histograms 

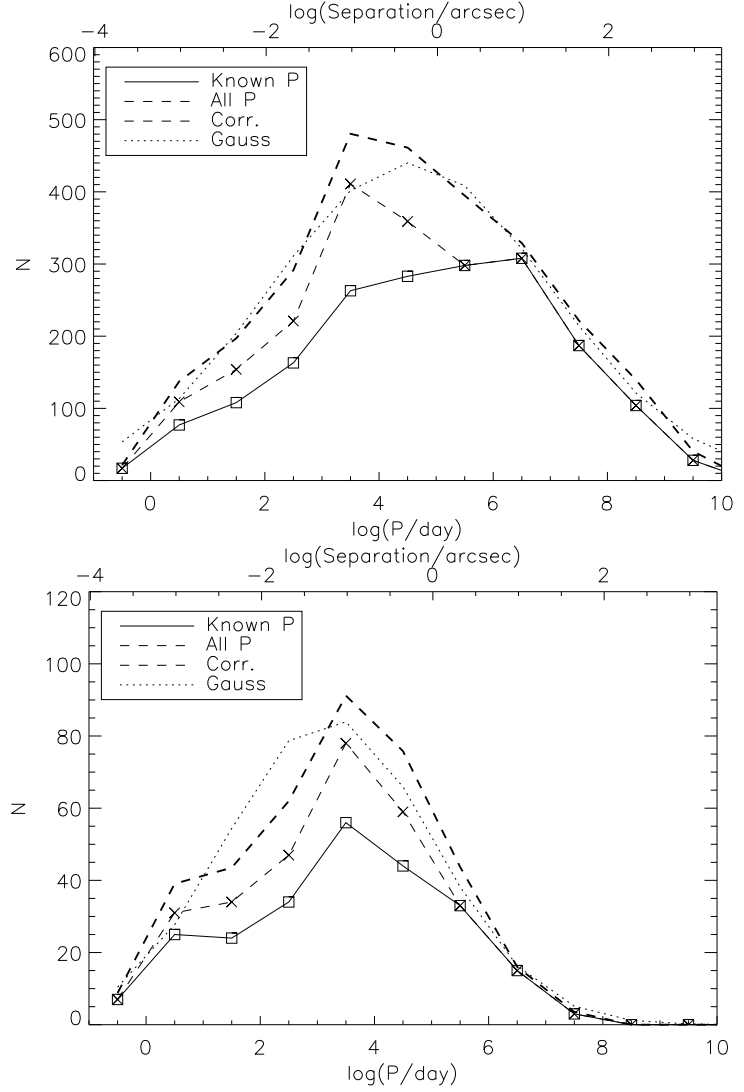

FIG. 6. - Histograms of periods in $\Delta x=1$ bins. Top: all pairs, bottom: level L11 only. In both plots, the full line is the distribution of pairs with known periods, the dashed lines adds fictitious periods, the thick dashed line corrects this distribution for selection, and the dotted line is the Gaussian distribution with parameters found by ML, normalized to match the total number of systems. Angular separation at a distance of $50 \mathrm{pc}$ is indicated on the upper horizontal axis of both plots.

in Figure 6 are indeed closer to straight lines than to a Gaussian.

Usually the distributions of binary parameters were studied regardless of hierarchy, merging all pairs together (DM91, R10). However, there is a significant (factor 2.5) difference between the median period of all binaries and the median period of outer binaries at level L1.

The periods of L11 sub-systems are shorter than the periods of all binaries, being restricted by the dynamical stability. The period histogram at L11 deviates from the Gaussian model, showing a depletion at $x \sim 2$ and an excess at $x<1$. Considering the uncertainty associated with the missing data, we cannot evaluate the significance of this mismatch. It is expected due to tidal migration of inner sub-systems towards short periods ( $\$ 4.4)$.

Note that the secondary sub-systems at L12 are only slightly less frequent that at L11. The common belief that inner systems are preferentially found around primary components turns out to be true, but this preference is not strong. The median mass of the primary components at levels L11 and L12 is $1.20 M_{\odot}$ and $0.79 M_{\odot}$, respectively.

The sub-systems at L12 show a tendency towards equal components, $\beta=1.3$. Only 5 secondary sub-systems out of 95 have unknown periods, making it difficult to explain high $\beta$ by the influence of the missing data. Considering the bias towards large $q$ in the detection of secondary sub- systems (see Figure 2), the high $\beta$ derived here for L12 should be verified by further work before being accepted. However, $\beta \sim 0$ would mean that more secondary subsystems are presently missed, so their frequency would be even higher than the frequency of L11.

\section{STATISTICS OF HIERARCHICAL SYSTEMS}

In this Section, we study statistical relations between binaries at different hierarchical levels. Are their periods or mass ratios related? Does the presence of one hierarchical level correlate with other levels? We propose a statistical model and test it by comparing simulations with the real sample.

\subsection{Period ratio and dynamical stability}

Figure 7 compares orbital periods $P_{S}$ and $P_{L}$ at the inner and outer hierarchical levels respectively (only known periods are plotted). It shows that the lowest period ratio $P_{L} / P_{S}$ is limited by the dynamical stability, but this ratio can also take large values; there is no typical or preferred period ratio. The points occupy almost all the space above the stability cutoff, showing that all allowed combinations of periods actually happen.

One striking feature of Figure [7] is the absence of outer periods shorter than $10^{3} \mathrm{~d}$, as noted already by Tokovinin et al. (2006). This is not caused by the observational selection, as tertiary companions with short periods are readily discovered by radial velocity variation; the detection probability at $x<3$ is high, see Figure 9 of Paper I. Gies et al. (2012) independently discovered the lack of short outer periods by eclipse timing of 41 binaries from Kepler: 14 of them show trends indicative of tertiary companions, but none has tertiaries with $P_{L}<700 \mathrm{~d}$. In the 3 -tier hierarchies L11+L111, the shortest period at L11 is $x=2.88$, confirming the exclusion zone of outer periods found for the L1+L11 and L1+L12 hierarchies. This said, triple systems with short outer periods are known among more massive stars (e.g. $\lambda$ Tau with $P_{L}=30 \mathrm{~d}$ ).

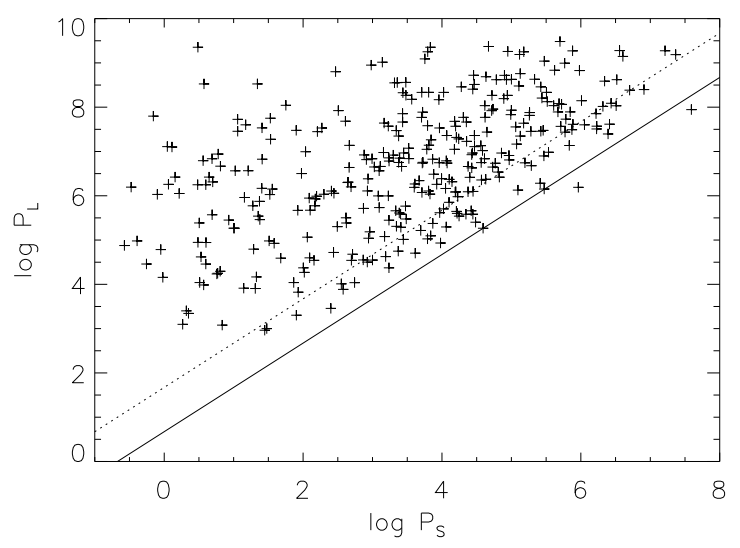

FIG. 7.- Orbital periods $P_{S}$ (short) at inner hierarchical levels L11 and L12 are compared to the periods of outer systems $P_{L}$ (long) at L1. The periods are expressed in days and plotted on the logarithmic scale. The solid and dotted lines mark $P_{L} / P_{S}$ of 4.7 and 47 , respectively (zone affected by the dynamical stability).

The minimum period (or separation) ratio allowed by dynamical stability has been studied by several authors. For example, the stability criterion of 


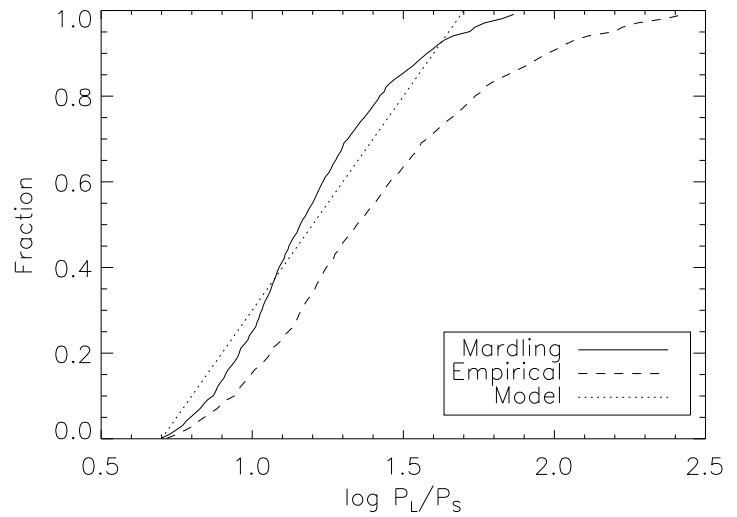

FIG. 8.- Cumulative distributions of the dynamical cutoff $P_{L} / P_{S}$ according to the equations (3) and (4) are compared to the adopted model of the dynamical truncation function $T$ (dotted line).

Mardling \& Aarseth (2001) for co-planar orbits can be written as

$$
P_{L} / P_{S}>4.7\left(1-e_{L}\right)^{-1.8}\left(1+e_{L}\right)^{0.6}\left(1+q_{\text {out }}\right)^{0.1},
$$

where $e_{L}$ is the eccentricity of the outer orbit, while the ratio of the distant-companion mass to the combined mass of the inner binary $q_{\text {out }}$ plays only a minor role. The solid line in Figure 7 corresponds to $P_{L} / P_{S}=4.7$; all points are above it (with two exceptions caused by the uncertainty of period estimates from projected separations). Tokovinin (2004) studied the dependence of the period ratio on $e_{L}$ for triple stars with reliable outer orbits and suggested an empirical stability limit with a stronger dependence on $e_{L}$,

$$
P_{L} / P_{S}>5\left(1-e_{L}\right)^{-3} \text {. }
$$

At $e_{L}=0.67$, the limiting period ratio (3) becomes a factor of 10 larger than at $e_{L}=0$. The dynamical truncation of $P_{L} / P_{S}$ is random and extends over at least one decade, depending on the distribution of $e_{L}$. Figure 8 shows the cumulative distributions of this cutoff $\Delta x=$ $\log _{10} P_{L} / P_{S}$ if a cosine distribution of $e_{L}$ between 0 and 0.8 is assumed, with average $e_{L}=0.4$. Available data on multiple stars indicate that orbits of outer systems tend to have moderate eccentricities (Shatsky 2001), the linear distribution $f\left(e_{L}\right)=2 e_{L}$ seems to be excluded.

Here we use a crude model for the dynamical truncation function $T(\Delta x)$ (probability of a triple system to be dynamically stable) by setting $T=0$ for $\Delta x<0.7$, $T=1$ for $\Delta x>1.7$, and linear in-between (dotted line in Figure 8). This model agrees with the stability criterion of Mardling \& Aarseth for the assumed distribution of $e_{L}$, while the criterion (4) predicts a stronger cutoff. An attempt to determine the truncation empirically by the distance of points in Figure 7 above the solid line did not produce any convincing results. Most outer and some inner periods are determined from projected separations to within a factor of 3 , so the empirical values of $\Delta x$ are approximate. For the same reason, precise knowledge of the truncation function $T(\Delta x)$ is not needed here and its crude model is sufficient.

\subsection{Independent multiplicity?}

The distributions of both inner and outer periods in the allowed region of Figure 7 look similar, as though they

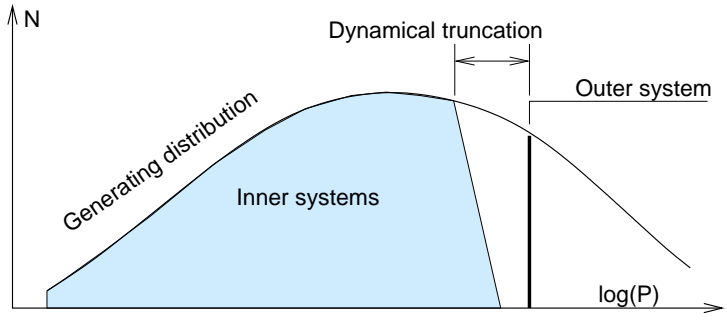

FIG. 9.- Explanation of independent multiplicity. The period of the outer binary is drawn from the log-normal generating distribution. Then the period of the sub-system is drawn from the left part of the same distribution fulfilling the stability constraint (the hatched part)

were drawn from the same population. Are the periods at levels L11 and L12 shorter than at L1 simply because of the dynamical cutoff?

The idea that the inner and outer pairs of multiple stars are selected from the same (or similar) parent distributions, subject only to the stability constraint, merits further investigation. Several arguments in its favor are furnished by prior work. Tokovinin et al. (2006) noted that the mass ratios of close binaries with and without outer (tertiary) companions are distributed equally, although the former group has statistically shorter periods. The frequency of spectroscopic sub-systems in visual binaries is similar to the frequency of spectroscopic binaries in the open clusters and field (Tokovinin \& Smekhov 2002). The frequency and mass ratio of resolved sub-systems in wide binaries are again comparable to the binaries of such separations in the field (Tokovinin, Hartung, \& Hayward 2010). Finally, the angular momentum vectors in the inner and outer systems show only a weak correlation (Sterzik \& Tokovinin 2002), and there are well documented cases of counterrotating multiples.

Independent multiplicity is expressed mathematically by setting the joint period distribution at levels L1 and L11 to be a product of the individual distributions at these levels, including the dynamical truncation factor $T(\Delta x)$ :

$$
f\left(x_{L 11}, x_{L 1}\right)=f_{L 11}\left(x_{L 11}\right) f_{L 1}\left(x_{L 1}\right) T\left(x_{L 1}-x_{L 11}\right) .
$$

Figure 9 illustrates this statistical model. The generating distribution for L11, $f_{L 11}\left(x_{L 11}\right)$, could be the same as the period distribution at L1, $f_{L 1}\left(x_{L 1}\right)$. This logic can be applied to other levels, leading to a complete statistical description of hierarchical multiplicity. Comparison with the real data presented below shows that this model is too simplistic and needs several adjustments. However, to the first order, it works even in its simplest form.

An attempt was made to test this idea using the ML method. The truncation function was added as a multiplier to the distribution (11) and the 4 parameters were found for the sub-systems at L11 and L12. The resulting distributions can be thought of as the generating functions $f_{L 11}$ and $f_{L 12}$. The problem of this approach is that almost all sub-systems have $x<6$ (see Figure 7), so the data do not constrain the generating functions at longer periods. This means that the fitted parameters of the generating distributions $\epsilon, x_{0}, \sigma$ do not inspire confidence, and only the distributions at $x<6$ (left halves of the Gaussians) are more or less defined. Moreover, a Gaussian distribution is not a good model for the sub- 


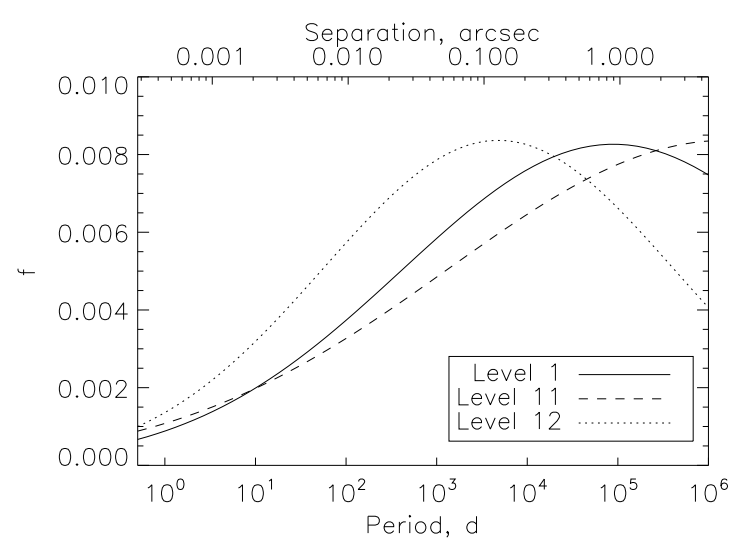

FIG. 10.- Comparison of the generating Gaussian distributions for levels L11 and L12 derived by ML with the period distribution of the outer systems at L1.

systems at L11 (Figure 6). We do not list here the fitted parameters and simply plot the relevant parts of the generating distributions in Figure 10, The periods of L12 sub-systems turn out to be shorter and their frequency at $x<4$ is even higher than the frequency of levels L1 and L11. However, considering the uncertainties involved in this crude model, we can only state that all three curves are not very different from each other and that selecting periods at outer and inner levels from the same distribution is not such a bad idea.

\subsection{Simulations}

In this sub-section, we simulate the multiplicity of the FG-67 sample. A synthetic population of single, binary, and multiple stars is created by a random draw from the generating distribution (11) with parameters $\left(x_{0}, \sigma, \beta\right)=$ $(5.0,2.3,0)$. The period distribution is truncated at $x<$ -0.3 and $x>10$. The binary frequency $\epsilon$ is specified below. For each component of a binary pair with $x>3$, the sub-systems are drawn from the same distribution and retained with a probability $T(\Delta x)$ defined above. The recipe is applied recursively to produce high-order hierarchies. After that, the synthetic sample is "filtered" by the average detection probability of the real sample (different for the primary and secondary components) and compared to the real sample.

Multiple systems with more than two hierarchical levels (3-tier hierarchy) pose a specific problem for their detection. Suppose that in a quadruple $(((\mathrm{Aa}, \mathrm{Ab}), \mathrm{B}), \mathrm{C})$ the component $\mathrm{B}$ is missed (un-detected). The system will be perceived as a triple $((A a, A b), C)$, i.e. we miss the intermediate hierarchical level; the pair $\mathrm{Aa}, \mathrm{Ab}$ is attributed to L11, instead of L111. Now suppose that the true system is $((\mathrm{A},(\mathrm{Ba}, \mathrm{Bb})), \mathrm{C})$ and we miss the same component B. In such case, we can't discover $(\mathrm{Ba}, \mathrm{Bb})$, the observed system becomes a simple binary (A,C). Our detection simulator eliminates all siblings of un-detected secondary components but keeps those of the primary, and corrects the hierarchical levels to reflect the observed, rather than true, structure of each system.

Straightforward application of the above recipe with a fixed $\epsilon$ over-produces binaries and under-produces triples, while the total number of L1 systems (the multiplicity fraction) is correct. Therefore, sub-systems at different hierarchical levels do have some correlation. The simulator is modified by adopting a variable binary fre- quency $\epsilon$ (however, $\epsilon$ is same for each individual multiple system). When $\epsilon$ is high, many hierarchical multiples are produced, and when it is low, we get mostly single stars and simple binaries. This reflects the idea that the field sample is a mixture of "binary-rich" and "binarypoor" populations. The average $\epsilon$ is constrained by the observed multiplicity fraction $f_{M}$, while the relative proportion of hierarchies informs us on the variation of $\epsilon$. The frequency of binary stars is proportional to $\epsilon$, the frequency of triples to $\epsilon^{2}$, etc. By adopting a variable $\epsilon$, we enhance the fraction of hierarchies until it matches the real sample (the same result could be obtained by increasing $\epsilon$ for inner hierarchical levels).

We split the simulated sample in three equal parts and apply to each group its own frequency $\epsilon_{i}$. The correct fraction of hierarchies was obtained, for example, for $\epsilon_{i}=[0.05,0.60,0.75]$. These numbers were found by trial and error, their mean 0.466 is the simulated multiplicity fraction. Compared to the constant multiplicity, the fraction of triples is increased by $\left\langle\epsilon^{2}\right\rangle /\langle\epsilon\rangle^{2}=1.41$. There are other ways to model variable $\epsilon$ by adopting some distribution and playing with its parameters. The distribution of $\epsilon$ chosen here is discrete with three equally probable values. Although the values of $\epsilon$ quoted above produce a good match to the data, other combinations or recipes are not excluded.

The simulator needs yet another adjustment to reproduce the large observed number of $2+2$ systems. If primary and secondary sub-systems at levels L11 and L12 are generated independently of each other, their common occurrence is too rare. The observed numbers of systems containing L11, L12, and both levels (i.e. $2+2$ systems) are $\left[N_{L 11}, N_{L 12}, N_{2+2}\right]=[296,95,41]$. We define the correlation $C=N_{2+2} / N_{L 12}=0.43$ as the fraction of $2+2$ systems among the L12 systems. Let $N_{L 1}=1747$ be the number of L1 (outer) binaries in the sample. If the occurrence and detection of L11 and L12 were mutually independent, their respective probabilities would be $N_{L 11} / N_{L 1}=0.169$ and $N_{L 12} / N_{L 1}=0.054$. The product gives an estimate of $2+2$ hierarchies as $N_{2+2}=16$, to be compared to the actual number $N_{2+2}=41$. The excess of $2+2$ systems indicates correlation between levels L11 and L12. This effect was noted by Tokovinin \& Smekhov (2002): the frequency of spectroscopic sub-systems in the distant tertiary components of known triples is enhanced in comparison to the field stars, as though the multiplicity syndrome were contagious. Sub-systems of levels L11 and L12 correlate because they were formed close to each other, in the same environment or even by the same process.

The excess of $2+2$ systems is accounted for in the simulations by increasing $\epsilon$ by $\epsilon_{+}=1.2$ times for the secondary sub-systems at L12, if the primary sub-system at L11 is already present. Otherwise, the frequency of L12 sub-systems is multiplied by $\epsilon_{-}=0.5$; without such reduction of $\epsilon$, the simulator over-produces the level L12. By playing with these two numbers, we adjust the total number of L12 systems and their correlation with L11. We also adopt $\beta=1.0$ for L12, guided by the ML results (when $\beta \sim 0$ is used for the secondary sub-systems, there are more undetected L12 pairs and the number of hierarchies becomes larger, $\left.f_{H}=0.15\right)$. We tried several other methods to simulate the observed correlation between L12 and L11, for example by modifying the periods 
TABLE 3

MUlTipliCity COUNTS IN REAL AND SIMULATED SAMPLES

\begin{tabular}{lrr|rrr}
\hline \hline Parameter & \multicolumn{2}{c|}{ Real } & \multicolumn{3}{c}{ Simulated } \\
& $N$ & $\langle x\rangle$ & $N_{\text {obs }}$ & $\langle x\rangle$ & $N_{\text {tot }}$ \\
\hline L1 & 1747 & 4.89 & 1776 & 4.91 & 2190 \\
L11 & 296 & 3.4 & 295 & 3.4 & 478 \\
L12 & 95 & 3.7 & 95 & 4.2 & 348 \\
L11 \& L12 & 41 & & 43 & & 196 \\
L111 & 17 & 2.1 & 23 & 2.5 & 49 \\
L112 & 4 & & 5 & & 33 \\
L121 & 3 & & 12 & & 36 \\
Single & 3053 & & 3024 & & 2610 \\
Binaries & 1397 & & 1428 & & 1560 \\
Triples & 290 & & 274 & & 366 \\
Quadruples & 55 & 62 & & 204 \\
2+2 quadruples & 37 & 35 & & 151 \\
Quintuples & 5 & 9 & & 43 \\
Hierarchies & 350 & 348 & \\
\hline \multicolumn{4}{c}{}
\end{tabular}

at L12 or by generating L12 with $\epsilon=1$ in presence of L11. The method chosen here gives good results, but it is not unique. Simulations give the observed correlation $C=0.45$ and the intrinsic correlation (before applying the detection filter) of 0.56 .

A reasonably good match between the real and simulated samples is demonstrated by Table 3. To reduce statistical errors, we simulated the sample $10 \times$ larger, $N=48000$, and scaled down the numbers. The simulated multiples successfully mimic the period distributions of the real sample and the plot in Figure 7 . The median periods at levels L1, L11, and L12 in the simulated and real samples agree well.

The last column of Table 3 gives the system count in the simulated sample before applying the detection filter. They represent the true multiplicity corrected for observational selection. Remember, however, that this method of correction depends on the simulation recipe used here, which is not unique. Although the simulated and real statistics match quite well, this does not mean that another equally good simulators leading to somewhat different predictions cannot be devised. Interestingly, the apparent number of pure binaries remains almost unaffected by the observational selection, while the numbers of true and observed hierarchies differ substantially, especially at level L12. The simulations predict that a large number $(\sim 150)$ of $2+2$ systems in the FG-67 sample remain to be discovered.

The simulator over-estimates the number of subsystems at high hierarchical levels L111, L121, L112, etc. because it does not account for tidal evolution in close inner binaries and selects their periods from the same log-normal distribution. We do not attempt to correct this effect and simply take it into consideration. Because of this, the simulated number of triples is smaller than observed (274 vs. 290), while the numbers of high-order hierarchies are larger. The companion frequency $f_{C}$ is slightly over-estimated. However, the total number of hierarchies, which depends only on levels L11 and L12 and on their correlation, is simulated correctly.

The total number of hierarchical systems in the simulated sample is 630 , leading to $f_{H}=0.132$. It agrees with $f_{H}=0.129$ estimated by the ML method (different experimental versions of the simulator produced $f_{H}$ between 0.12 and 0.15$)$. The fractions of L11, L12,

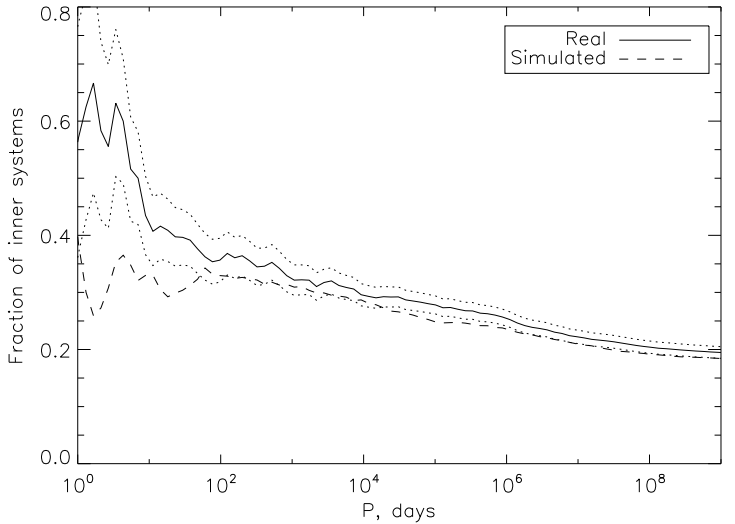

FIG. 11. - The observed fraction of inner sub-systems (levels $>$ L1) among all pairs up to a given period is plotted in the full line. The dotted lines show the statistical errors, the dashed line corresponds to the simulated sample.

and $2+2$ systems in the simulated sample are 0.100 , 0.073 , and 0.041 , respectively. The estimated fractions of single, binary, etc. systems (last column of Table 3) are $f_{n}=[0.543,0.323,0.076,0.043,0.013]$, where the last number is the fraction of systems with 5 or more components. The calculated companion fraction is then $f_{C}=0.65$. As expected, it is larger than its ML estimate $\epsilon=0.57$ (the first line in Table 2). The actual companion fraction must be about 0.6.

It is truly remarkable that complex statistics of hierarchical multiple systems can be reproduced by the simulation recipe with only five adjusted parameters $\epsilon_{i}, \epsilon_{+}$, and $\epsilon_{-}$, while the remaining parameters of the generating distribution $x_{0}, \sigma, \beta$ are determined by the ML method and kept fixed. This supports the underlying idea of independent multiplicity. Therefore, the shorter periods of sub-systems at levels L11 and L12 (compared to all binaries) can be explained by the dynamical truncation alone.

\subsection{Tidal evolution}

Figure 11 illustrates one aspect of the data that is not yet captured by the simulator. All binaries up to a certain period $P$ are selected, and the observed fraction of inner pairs in multiples (levels higher than L1) among those binaries is plotted. The fraction of inner systems reaches $\sim 0.6$ at $P<3 \mathrm{~d}$ and slowly drops to 0.18 with increasing period. The fraction of inner pairs in the simulated sample (with the detection filter applied), plotted in dashed line, is quite similar to the observed one, except at $P<10 \mathrm{~d}$ where it is much lower.

Inner binaries in triple systems with mutually inclined orbits experience periodic modulation of their eccentricity by the Kozai-Lidov cycles. When the separation at periastron becomes comparable to the stellar radii, tidal friction absorbs the orbital energy and the period of the inner binary shortens to $P_{S} \lesssim 10 \mathrm{~d}$. This mechanism, known as Kozai cycles with tidal friction (KCTF, Eggleton 2006), works well when the period ratio $P_{L} / P_{S}$ is not too high and the initial inner period $P_{S}$ is not too long. It causes migration of inner periods from $P_{S} \sim 100$ d to $P_{S} \leq 10$ d (Fabrycky \& Tremaine 2007), while some inner binaries can merge.

Tidal migration of inner periods reflects in their period distribution (lower panel in Figure 6), although the 


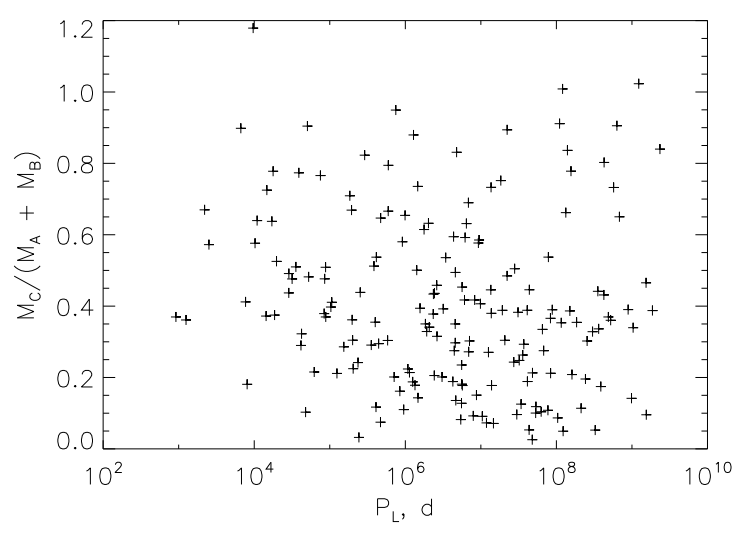

Fig. 12. - System mass ratio $q_{\mathrm{sys}}=M_{C} /\left(M_{A}+M_{B}\right)$ in the outer (level L1) systems of triple stars as a function of the outer orbital period $P_{L}$.

addition of unknown fictitious periods to the histogram partially masks the effect. See also the cumulative period histogram at L111 in Figure 3, where $40 \%$ of periods are shorter than $\sim 3 \mathrm{~d}$. The period distribution of spectroscopic binaries in the Hyades (Griffin 2012, figure 68) clearly shows the depletion at $P>10 \mathrm{~d}$ and the peak at shorter periods. The non-monotonous period distribution was also found by Halbwachs et al. (2003). Tidal evolution explains why the median period at L111 is shorter that its simulated value and why the simulator over-estimates the number of high hierarchical levels. Apparently, some close inner binaries merged.

\subsection{Mass ratios in hierarchical systems}

We examined correlations between the mass ratios in triple systems. The 199 simple triples (levels L11 or L12 without further inner sub-systems, with known periods and masses) are used. The data are affected to some extent by the observational selection, as no simple correction can be suggested in this case. We found no correlation between the mass ratios in inner and outer systems and between the mass ratio and the period. Similar conclusion was reached by Tokovinin (2008) from analysis of the biased catalog. For example, Figure 12 plots the ratio $q_{\text {sys }}$ of the distant companion's mass to the mass sum of the inner binary. The median value of $q_{\text {sys }}$ for outer binaries is 0.39 , with no obvious dependence on the outer period $P_{L}$ (when the sample is split in two halves at $P_{L}=10^{6.7} \mathrm{~d}$, the $q_{\text {sys }}$ medians are 0.40 and 0.39 ). The median mass of the tertiary components is $0.73 M_{\odot}$, the median mass of the secondary components in all binaries is $0.70 M_{\odot}$. Therefore, there is no evidence that the distant tertiary components are less massive than the components of the inner binaries.

Tokovinin (2008) noted that in $2+2$ quadruples with $P_{L}<10^{5.4} \mathrm{~d}$ both inner binaries have similar total masses and periods. The FG-67 sample contains only 5 such quadruples, all with $q_{\text {sys,out }}>0.5$. The statistics are too small to make any further conclusions.

We checked whether twin binaries have any preference to be found in multiple systems. Half of the 96 binaries with $P<10 \mathrm{~d}$ are inner sub-systems (see Figure 11). Among those 96 binaries, 18 are twins with $q>0.95$, and 8 of those twins (also a half) are in inner sub-systems. Therefore, the phenomenon of twin binaries is not directly related to the hierarchical multiplicity. The mass

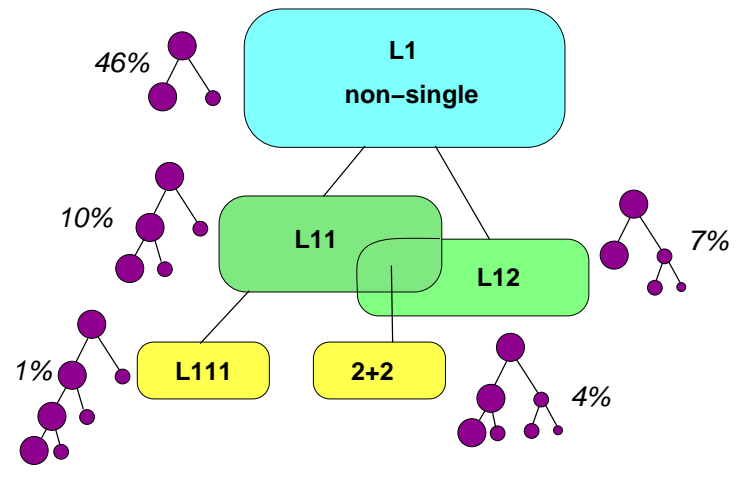

FIG. 13. - Fractions of different hierarchies in the FG-67 sample. The levels L11 and L12 overlap by $4 \%$, accounting for the $2+2$ systems, while the total fraction of hierarchies is $10+7-4=13 \%$.

ratios of all binaries, including twins, seem to be independent of their hierarchical level.

One notable exception to the above statement concerns secondary sub-systems at L12 which show a tendency to equal masses, $\beta=1.3$, according to the ML parameter-fitting $(\S$ 3.3). About half of those binaries (41 out of 95) also have primary sub-systems at L11. The median mass ratio in those 41 primary sub-systems is 0.75 , while the remaining majority of L11 sub-systems have median mass ratio of 0.65 . This result does not rely on the ML analysis or completeness correction, it is a straightforward comparison between the primary subsystems whose outer companions are themselves either binary or single. Preference of equal masses is thus typical for both inner pairs in $2+2$ hierarchies. This suggests that they could have formed differently, compared to other binary and triple stars.

\section{SUMMARY AND CAVEATS}

The main results of this study are:

1. We confirm known facts about binary statistics of solar-type stars. Periods of all binaries (regardless of their hierarchy) are distributed log-normally with a median $x_{0}=4.54(\sim 100 \mathrm{yr})$ and dispersion 2.4. The mass ratio is distributed uniformly and is independent (or almost independent) of the period, except such details as short-period twins. The multiplicity fraction is $0.46 \pm 0.01$.

2 . The fraction of hierarchical systems is $0.13 \pm 0.01$, of which 0.10 have sub-system(s) related to the primary component, 0.07 have sub-systems in the secondary component, and 0.04 have both (Figure13). The presence of sub-systems in both components of wide (outer) binaries is correlated.

3. There remain about 150 undiscovered secondary (L12) sub-systems. About $60 \%$ of those pairs reside in $2+2$ hierarchies, because of the above correlation.

4. The lack of outer systems with periods shorter than $\sim 1000 \mathrm{~d}$ is real (not a selection effect).

5. The statistics of hierarchical systems can be reproduced by recursive random selection of outer and inner binaries from the same generating period distribution with parameters $x_{0}=5.0, \sigma=2.3$, and 
uniform mass ratio, $\beta=0(\beta=1.0$ for the secondary sub-systems). The amplitude of this distribution is variable (e.g. $\epsilon=[0.05,0.60,0.75]$ with equal probability). Only dynamically stable combinations are kept, with the dynamical cutoff in $\log \left(P_{L} / P_{S}\right)$ uniformly distributed between 0.7 and 1.7. The frequency of secondary sub-systems is enhanced by their correlation with the primary subsystems, while binaries with periods shorter than $1000 \mathrm{~d}$ do not produce sub-systems.

6. The period distribution in the inner sub-systems can be explained by dynamical truncation. However, there is an excess of tight inner binaries with $P<10$ d compared to the smooth Gaussian distribution, presumably caused by tidal evolution.

7. The mass ratios in the inner and outer systems of triple stars are uncorrelated, except $2+2$ hierarchies where the mass ratios are larger than average in both of their inner sub-systems. In triple stars, the system mass ratio of the outer binary does not depend on its period and has a median value of 0.39 , meaning that the masses of tertiary components are comparable to the masses of stars in the inner binary.

The above results are not free from biases, approximations, and errors. Some were mentioned above and in Paper I. We re-capitulate the most relevant weaknesses below, in order of decreasing importance.

1. Missing information. Unknown periods and mass ratios of many spectroscopic and astrometric binaries (32\% of all binaries with $P<100 \mathrm{yr}$ ) increase the uncertainty of the results. Missing information is accounted for in the ML analysis and in the simulations. However, we cannot reliably study the distribution of periods and mass ratios of close binaries until they are resolved or get spectroscopic orbits.

2. Approximate knowledge of detection probability. Despite the effort to collect relevant data and to model the detection limits, there remains some uncertainty. For example, all binaries with separation of $\sim 1^{\prime \prime}$ and $\Delta V<4$ are assumed to be resolved by Hipparcos, but this is not actually the case. The detection of sub-systems in the secondary components is most critical, as it entails a larger correction for incompleteness.

3. Uncertain data and subjective decisions. Some binaries accepted as real may in fact be spurious or optical. Conversely, we may have rejected or missed some real pairs (see $\S 3.10$ of Paper I). The percentage of such cases is certainly much less than $10 \%$ of all binaries, but we do not know how much less.

4. Biases against multiple stars. Secondary components that are themselves binary can be partially resolved and, for this reason, be missed by the point-source catalogs. Their proper motion may differ substantially from that of the main target.
The Hipparcos parallaxes of unrecognized binaries are often biased by their orbital motion, relegating some of them beyond the $67 \mathrm{pc}$ distance limit. Such nearby multiples as $\zeta$ Cnc and $\xi$ UMa are missed here because they are not included in the Hipparcos catalog.

5. White dwarfs. The fraction of Sirius-like binaries harbouring a WD companion is estimated as $\sim 2 \%$. Known Sirius-like systems are removed from the analysis, but the reduction of the sample size associated with the removal of WDs is uncertain. Most likely, the assumed sample size $N_{t o t}=4800$ should be reduced further, the estimated $f_{M}$ will then increase slightly.

6. Approximations made in the data analysis: relation between mass and absolute magnitude in various photometric bands, evaluation of period from projected separations, evaluation of the mass ratio from photometry or from the minimum mass of single-lined spectroscopic binaries, distribution of periods and mass ratios approximated by analytical functions.

It is clear that the results of this study are affected by these biases and that the formal errors quoted above underestimate the actual, larger uncertainties. Future work will revise some of the parameters derived here. However, these anticipated changes will no longer be dramatic; the fraction of hierarchical systems derived here should not be revised by more than $1-2 \%$.

\section{DISCUSSION}

\subsection{Comparison with previous work}

The large sample of solar-type binaries with known detection limits confirms and strengthens known facts about their statistics (Duchêne \& Kraus 2013). The parameters of the log-normal period distribution determined here $\left(x_{0}=4.54, \sigma=2.40\right)$ match well the results of $\operatorname{R10}\left(x_{0}=5.03, \sigma=2.28\right)$. The triangular function (2) fits the distribution of $x=\log P$ even better than a Gaussian. However, the period distribution of the inner sub-systems is not log-normal, it has an excess at $P<10 \mathrm{~d}$

We find a nearly flat distribution of the mass ratio with the power index $\beta \sim 0$ and show that it does not depend on the orbital period (with a caveat related to partially missing binary parameters). Duchêne \& Kraus (2013) fitted $\beta=0.28 \pm 0.05$ to the data of R10, but found that $\beta=1.16 \pm 0.16$ for periods shorter than $10^{5} \mathrm{~d}$. Considering the sensitivity of derived $\beta$ to the treatment of selection effects (see $\S 3.2$ ), we believe that this claim is premature. Reggiani \& Mever (2013) derive $\beta=0.25 \pm$ 0.29 for the two combined samples of dwarf binaries and argue for the independence of $\beta$ on period and primary mass. The flat $f(q)$ was found by Kraus et al. (2011) for the pre-main-sequence stars in Taurus more massive than $0.7 M_{\odot}$.

This study gives the first look at unbiased statistics of hierarchical systems with three or more components. Multiples are frequent (one in eight stars). The $13 \%$ frequency of hierarchies estimated here is essentially the same as $12 \%$ found by Raghavan et al. (2010) in the 
25-pc sample. However, they focused on companions to the primary stars and missed some secondary pairs. A few of those secondary sub-systems are recovered here, more are yet to be discovered. On the other hand, these authors may have over-estimated the number of highorder multiples. They list three multiples with five or more components (their Fig. 24), while we found only 5 such systems in a sample $10 \times$ bigger. This discrepancy is explained by the better companion census of nearby stars, by the inclusion of uncertain sub-systems (such as HD $186858 \mathrm{Aa}, \mathrm{Ab}$ ) in the 25-pc sample, and by the small-number statistics.

Note that among the 11 quadruples in the 25-pc sample, 9 (or $82 \%$ ) have the $2+2$ hierarchy and only two have the $3+1$ hierarchy. The last column of Table 3 predicts a $74 \%$ fraction of $2+2$ quadruples, in agreement with the small statistics within $25 \mathrm{pc}$.

Fuhrmann (2011) gives estimates of $f_{M}$ and $f_{H}$ among F-type stars within 25 pc that agree well with our results. He claims that both fractions rise sharply with increasing primary mass (however, with a low significance limited by the sample size). We do not confirm this trend: the observed (not selection-corrected) multiplicity fraction of 845 stars with $M>1.3 M_{\odot}$ within $67 \mathrm{pc}$ is $38 \pm 2 \%$, not significantly different from $36 \%$ in the full FG-67 sample.

\subsection{Statistical model of multiplicity}

The statistics of hierarchical multiples are well modeled by independent selection of sub-systems from some generating distribution of periods, provided that the binary frequency is variable. This hints that the field stars were formed in different environments, binary-rich and binary-poor. Indeed, the binary frequency differs between star-forming regions (King et al. 2012). Moreover, some fraction of single stars come from disintegrated multiples and merged binaries, so the intrinsic frequency of the formation process $\epsilon$ is always larger than the multiplicity of a mature field population.

Variable multiplicity naturally leads to the enhanced probability of finding sub-systems in wide binaries, in comparison to all targets. This tendency was noted by Makarov et al. (2008). The simulations faithfully reproduce the fraction of close binaries belonging to higherorder systems for the inner periods longer than $10 \mathrm{~d}$ (Figure 11); at shorter inner periods, the observed fraction is much higher than predicted, because of tidal evolution.

Kraus et al. (2011) already tried to model hierarchical multiplicity in a Monte-Carlo simulation by producing inner sub-systems with the generating period distribution and applying the stability cutoff. Similar approach was taken by Santerne et al. (2013) in their modeling of hierarchical multiples for evaluation of false positives in exo-planet detection. The multiplicity simulator developed here is anchored to the data and can be used in such studies with confidence.

\subsection{The $2+2$ systems}

Correlated presence of sub-systems in both components of a wide binary is the new fact found in the course of this project (Reed et al. 2014). To reproduce this correlation by simulation, the frequency of secondary sub-systems is enhanced in presence of the primary subsystem and decreased otherwise. As a result, the majority of quadruple systems have the $2+2$ hierarchy $(67 \%$ observed, $74 \%$ predicted after correction of observational selection, $82 \%$ in the $25-\mathrm{pc}$ sample). The fraction of quadruple stars is then larger than predicted by the independent multiplicity, and comparable to the fraction of triples (4\% and $8 \%$, respectively). The multiplicity fractions $f_{n}$ do not decrease in geometric progression of $n$, as suggested in the early works (Batten 1973) and repeated by Duchêne \& Kraus (2013). Poor detection of sub-systems in secondary components was not fully appreciated until recently.

The relatively large fraction of $2+2$ systems suggests that they were formed by some special process. Other properties of $2+2$ quadruples such as similarity of component's masses and inner periods point in this direction, too (Tokovinin 2008). Both inner sub-systems in the $2+2$ hierarchies differ from the rest of inner sub-systems by their larger mass ratios $(\S[4.5)$. On the other hand, when only one inner sub-system is present, it is preferentially associated with the most massive star, and its mass ratio is distributed uniformly, as in normal binaries. Therefore, there must be another, dominant formation channel which produces most binary and triple stars and, by extension, $3+1$ quadruples.

\subsection{Implications for multiple-star formation}

Statistical work on multiple stars, including this one, is motivated by the desire to understand their formation. What have we learned from this study?

Hydrodynamical simulations (Bate 2012) reveal a complex process of binary formation and early evolution that shapes the multiplicity statistics. Stellar pairs form by fragmentation with initial separations larger than 10100 AU (so-called opacity limit) and small masses. The components grow by accreting gas and, at the same time, migrate to smaller separations. The depletion of long periods (the right side of the "Gaussian") by dynamical interactions with the environment, combined with migration, shape the observed period distribution (Korntreff et al. 2010). If the fragmentation generates periods $x=\log P$ longer than, say, $x=4$ (a step function in $x$ ) and the period decay $\Delta x$ is a random function (e.g. uniformly distributed), the convolution of those two distributions results in the linear decrease of binary frequency at $x<4$, as actually observed (Figure 6). The approximately linear decrease of the period distribution at $x<4$ is produced under a wide range of assumptions, provided only that the initial period distribution has a lower cutoff and that the migration is random.

Migration is essential in understanding the origin of tight hierarchical systems where 3 or 4 stars are packed in a small volume. If all inner binaries formed with initial periods $x \geq 4$, the initial outer periods must be even longer, whereas the present-day outer periods can be as short as $x=3$ (Figure 7). Migration occurs therefore at all hierarchical levels. The inner binaries must migrate faster or form earlier than the outer binaries, otherwise the multiple system becomes dynamically unstable and decays.

Most hierarchies seem to be assembled "from insideout", by bringing together pre-fabricated inner pairs (or stars) to make the outer system. The success of modeling the observed statistics by independent multiplicity strongly supports this view. The mass ratios of inner and outer systems do not correlate, the masses of outer (ter- 
tiary) components are comparable to the masses of the inner components. The inner and outer periods are selected independently from the same distribution and are mutually related only by the dynamical stability (taking aside the tidal evolution). If hierarchies formed "from outside-in" by successive fragmentation of outer binaries, as envisioned e.g. by Kraus et al. (2011), it would be difficult to explain the independent multiplicity.

Considering that many hierarchies are close to the stability limit, dynamical decay of multiple systems must occur sometimes. It can happen when the outer binary migrates faster than the inner one or when other stars in the cluster disrupt the multiple or exchange components with it. However, multiple systems produced by $N$-body dynamical interactions (or surviving them) have characteristics that do not match real hierarchies: high eccentricities, low masses of distant components, and moderate ratios $P_{L} / P_{S}$. The observed multiplicity statistics indicates that dynamical processes play only a secondary role. Most hierarchical stellar systems in the field are not the surviving remnants of primordial clusters.

The $2+2$ hierarchies could be formed in a special way, e.g. by inelastic collision of two cores that prompts their fragmentation into sub-systems and, at the same time, keeps these two pairs together on a common wide orbit by dissipating the energy. Such impulsively-triggered multiple-star formation was envisioned by Whitworth (2001), see his Figure 2. Further evolution will depend on the relative orientation of the inner and outer orbits. If they are not aligned, the inner binaries will accrete gas with a misaligned angular momentum, which will accelerate their migration to short periods. In the opposite case of co-aligned orbits, the accretion will keep the inner binaries wide, and a quadruple with a moderate $P_{L} / P_{S}$ ratio and nearly co-planar orbits, resembling $\varepsilon$ Lyr, can be formed. Another striking aspect of this prototypical $2+2$ quadruple - similar masses of all 4 stars - matches the tendency to equal component masses in both inner sub-systems of $2+2$ hierarchies, emerging from this study.

On the other hand, the majority of the inner subsystems have $\beta \sim 0$, like all binaries, while their tertiary components are single. Their formation process should therefore be different from the formation of $2+2$ hierarchies. The tertiary component could have formed around the initial binary (or joined it) at a later stage. The cascade can continue further by adding yet another star in a $3+1$ hierarchy.

The $2+2$ formation channel probably produced more quadruples than actually observed because some subsystems merged, leaving triples and binaries, while some quadruples decayed dynamically. A certain fraction of binaries and triples in the field could be born as $2+2$ systems. Also, merging of the inner binaries (natural extension of migration) can possibly help in understanding why the outer periods in triple stars are longer than $10^{3} \mathrm{~d}$.

It should be stressed that not all close binaries belong to multiple stars. This is true only for pairs with $P<3$ d which could not be produced without tidal migration during their main-sequence life. At longer periods $P \geq 10 \mathrm{~d}$, the frequency of wide tertiary companions is comparable to the frequency of such companions in the full sample (Tokovinin et al. 2006).

Figure 14 illustrates the formation channels and the

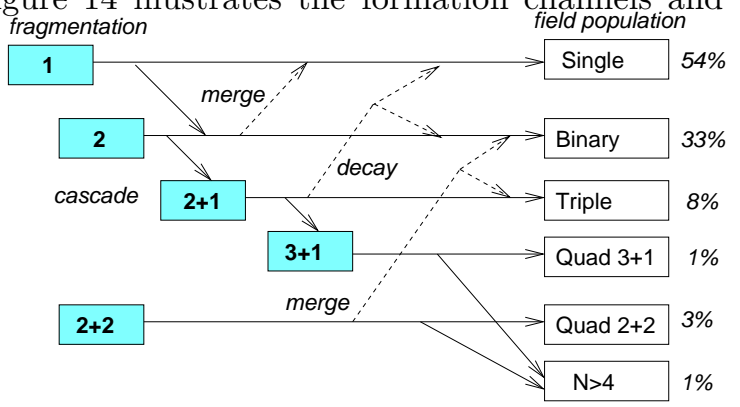

FIG. 14.- Formation channels of multiple systems and fractions of different hierarchies in the field.

fractions of different hierarchies in the field. The fragmentation produces single and binary stars. Cascade "inside-out" fragmentation generates $2+1$ triples and $3+1$ quadruples. Unstable hierarchies decay dynamically into single and binary stars, some close binaries merge. The alternative $2+2$ process creates quadruple stars with components of similar mass. Merging of inner pairs in the $2+2$ quadruples contributes to the populations of triples and binaries. About $1 \%$ of targets with more than 4 components could be produced by the combination of these elementary processes.

\subsection{Concluding remarks}

There are various ways to improve and continue the present study. The determination of unknown periods and mass ratios of close binaries is one obvious project to pursue. The prediction of the large number of undiscovered sub-systems in the secondary components should be tested observationally. It will be interesting to study relative motions in resolved triple and quadruple systems to get a better idea of the relative orbit orientation.

The data used in this work are acknowledged in Paper I. I thank Bo Reipurth and M. Bate for comments on the draft of this paper, P. Eggleton for discussions of multiplicity statistics and white dwarfs.

\section{APPENDIX}

\section{MAXIMUM LIKELIHOOD FORMALISM}

We want to model the data - periods and mass ratios of binaries - by an analytical distribution function $f(x, q)$ such as equation 1 i.e. find the best-fitting parameters of this model and their errors. Some data are missing (unknown periods and mass ratios).

Let the sample of $N$ targets contain $K$ known binary systems with period logarithms $x_{k}$ and mass ratios $q_{k}$. For each target (binary or single) we also estimate the companion detection probability $d_{i}(x, q)$. We maximize the likelihood function $\mathcal{L}$ - the probability of getting the actual data, given the model parameters. The maximum likelihood (ML) approach used here is equivalent to the Bayesian method (Allen 2007) with uniform priors.

For each $i$ th star, the probability $p_{i}$ follows the Poisson distribution $p(m)=(\mu / m !) \exp (-\mu)$ with parameter $\mu_{i}$ 
(probability of a detectable companion) and the integer argument $m=0$ for single stars and $m=1$ for binaries. The stars are observed independently of each other, so $\mathcal{L}$ is the product of $p_{i}$. Instead of maximizing $\mathcal{L}$, we minimize its natural $\log$ arithm $S=-2 \ln \mathcal{L}$. Elementary derivation leads to

$$
S=2 \sum_{i=1}^{N} \mu_{i}-2 \sum_{k=1}^{K} \ln \mu_{k} .
$$

The summation over $k$ extends only over detected companions. The first term equals $N \mu_{0}, \mu_{0}$ is the average probability of companion detection. The probabilities for the binary companions are $\mu_{k}=f\left(x_{k}, q_{k}\right) d_{k}\left(x_{k}, q_{k}\right)$, while for all targets $\mu_{i}$ in the first term are the products $f(x, q) d_{i}(x, q)$ averaged over the considered part of the parameter space $(x, q)$. Any normalization factor in the probabilities $\mu_{k}$ for binaries that is independent of the parameters has no influence on the result.

For binaries with known periods but unknown secondary masses, the products $f\left(x_{k}, q\right) d_{i}\left(x_{k}, q\right)$ are averaged over $0<q<0.8$. The rationale is that binaries with $q>0.8$ would have been recognized as double-lined or resolved, therefore the unknown mass ratios are somehow constrained. Binaries where both period and mass ratio are unknown have the detection probabilities averaged over $q<0.8$ and also over periods, assuming $x<4.5$. It is important to include both kinds of binaries with partial information in the calculation, as they are actually discovered.

For each binary, the detection probabilities are computed on the 2-dimensional grid with 100 points in $x,-0.3<$ $x<10$, and 20 points in $q$. Calculation of detection probability for the secondary components is done by a different program, as explained in Paper I, and saved in a different array of the same dimension. The distribution function $f(x, q)$ is computed on the same grid, the first bin in $q$ is set to zero (no companions with $q<0.05$ ). The normalization constant $C$ in equation (10) is computed numerically.

The ML formalism was tested on the simulated samples. Here the model and the sample match by definition, and the ML method indeed recovers the true parameters of the distributions, even when a realistic fraction of binaries is assumed to have unknown periods and mass ratios, as in the real sample.

For calculation of the generating distributions at levels L11 and L12, the ML algorithm was modified to include the dynamical truncation function $T(\Delta x)$. For calculating the mass-ratio distribution in selected intervals of period, the detection probabilities are averaged over these intervals, leaving only their dependence on $q$. Then only two parameters $\epsilon, \beta$ are fitted to the data.

The contours of $S$ in the parameter space define confidence limits: $\Delta S=1$ corresponds to the $68 \%$ interval $(1 \sigma)$, $\Delta S=2.71$ to $90 \%$, etc., in direct analogy with the Gaussian probability distribution. The error of some parameter $\xi$ is computed from the one-dimensional function $S(\xi)$, while other parameters are fixed. This is correct if the parameters are not correlated. We checked the absence of strong correlation by plotting the contours of $S$ while varying any two parameters around their best values. The contours are more or less round, indicating the lack of significant correlation between the parameters.

The ML method appears more rigorous than it actually is. Its systematic errors (biases) are caused by the mismatch between the actual data and their model (both the parametrized distribution and the detection probabilities). The confidence intervals returned by the ML describe only the statistical errors and do not account for these systematic errors. Confidence in the ML results for the real sample is gained by checking robustness with respect to slight changes in the models, alternative probability calculation, application to sub-samples, etc.

\section{REFERENCES}

Abt, H. A., \& Levy, S. G. 1976, ApJS, 30, 273 (AL76)

Allen, P. R. 2007, ApJ, 668, 492

Bate, M. R. 2012, MNRAS, 419, 3115.

Batten, A. H. 1973, Binary and multipls systems of stars (Oxford: Pergamon)

Duchêne, G. \& Kraus, A. 2013, ARAA, 51 (ArXiv:1303:3028)

Duquennoy, A. \& Mayor, M. 1991, A\&A, 248, 485 (DM91)

Eggleton P., 2006, Evolutionary Processes in Binary and Multiple Stars. Cambridge Univ. Press, Cambridge, UK

Eggleton, P. P. 2009, MNRAS 399, 1471

Fabrycky D. \& Tremaine S., 2007, ApJ, 669, 1298

Fekel, F. C. 1981, ApJ, 246, 879

Fuhrmann, K. 2011, MNRAS, 414, 2893

Gies, D. R., Williams, S. J., Matson, R. A. et al. 2012, AJ, 143, 137

Griffin, R. F. 2012, JAA, 33, 29

Halbwachs, J L., Mayor, M., Udry, S. \& Arenou, F. 2003, A\&A, 397, 159

Harrington, R. S. 1972, Celest. Mech., 6, 322

King, R. R., Parker, R. J., Patience, J., \& Goodwin, S. P. 2012, MNRAS, 421, 2025

Korntreff, C., Kaczmarek, T., \& Pfalzner, S. 2012, A\&A, 543, 126

Kraus, A. L., Ireland, M. J., Martinache, F., \& Hillenbrand, L. A. 2011, ApJ, 731, 8

Kuiper, G. P. 1935, PASP, 47, 121
Lucy, L. B. 2006, A\&A, 457, 629

Makarov, V. V., Zacharias, N. \& Hennessy, G. S. 2008, ApJ, 687, 566

Mardling, R. A. \& Aarseth, S. J. 2001, MNRAS, 321, 398

Raghavan, D., McAlister, H. A., Henry, T. J. et al. 2010, ApJS, 190, 1 (R10)

Reed, R., Tokovinin, A., Mason, D. B. et al, 2014, AJ, in preparation.

Reggiani, M. \& Meyer, M R. 2013, A\&A, 124

Reipurth, Bo, Boss, A. P., Clarke, C. J. et al. Multiplicity in early stellar evolution. In: Protostars and Planets VI, 2014.

Santerne, A., Fressin, F., Díaz, R. F. et al. 2013, A\&A, 557, 139 Shatsky, N. 2001, A\&A, 380, 238

Sterzik, M. \& Tokovinin, A. 2002, A\&A, 384, 1030

Tokovinin, A. A. 1992, A\&A, 256, 121

Tokovinin, A. 1997, A\&AS 124, 75

Tokovinin, A. A. 2000, A\&A, 360, 997

Tokovinin, A. A. \& Smekhov, M. G. 2002 A\&A, 382, 118

Tokovinin, A. 2004, Rev. Mex. Astron. Astrof., Conf. Ser., 21, 7

Tokovinin, A., Thomas, S., Sterzik, M., \& Udry, S. 2006, A\&A, 450,681

Tokovinin, A. 2008, MNRAS, 389, 925

Tokovinin, A., Hartung, M., \& Hayward, Th. L. 2010, AJ, 140, 510

Tokovinin, A. 2011, AJ, 141, 52 
Tokovinin, A. From binaries to multiples I: Data on F and G

dwarfs within 67 pc of the Sun. 2014, AJ, submitted (Paper I).

Whitworth, A. P. 2001, in: Proc. IAU Symp. 200, eds. H.

Zinnecker \& R. D. Mathieu (ASP: San Francisco). 